\title{
Antimicrobial Evaluation of Copper Oxide Nano-particles Synthesized Using Leaf Extract of Terminalia chebula
}

\author{
Deepika Sharma, Nutan Thakur, Jitendra Vashistt, Gopal Singh Bisht * \\ Department of Biotechnology \& Bioinformatics, Jaypee University of Information Technology, Waknaghat, Solan, Himachal Pradesh, \\ India- 173234
}

Keywords

Terminalia chebula; Zeta Potential;

Kirby Bauers's

Disk Diffusion;

Mueller Hinton.

Address for Correspondence: Gopal Singh Bisht, bisht.gopal@juit.ac.in

\begin{abstract}
Metallic nano-particles have high surface area to volume ratio making them a good candidate for biological applications. Metallic nano-particles can be prepared by chemical as well as green synthesis approach. Nowadays, green synthesis approaches are preferred over chemical synthesis as they minimize the use of harmful solvents. Present study focuses on evaluating antibacterial activity of nano-particles synthesized by facile green chemistry approach against gram negative pathogens. Keeping this in mind, we have synthesized copper oxide nano-particles from Fehling's solution A \& B using Terminalia chebula. Synthesis of metallic nano-particles was confirmed by UV spectroscopy. They were further characterised by dynamic light scattering technique in order to determine their zeta potential and size distribution values. The antibacterial spectrum of nano-particles was determined against standard bacterial strains; Escherichia coli ATCC 25922 and Acinetobacter baumannii ATCC 19606 using Kirby Bauer's disk diffusion on Mueller Hinton agar. Results of AST were interpreted according to CLSI and ICMR guidelines for compound and standard antibiotic disc. This is the first report on synthesis of copper oxide nano-particles using aqueous extract of T. chebula leaves. Present study revealed that copper oxide nano-particles can be easily synthesized using natural reducing agents present in leaves of T. chebula. Synthesized metallic nano-particles were found to have stable zeta potential values and size less than $370 \mathrm{~nm}$. Copper oxide nano-particles inhibited visible growth of bacteria at concentrations ranging between $125 \mu \mathrm{g}-250 \mu \mathrm{g}$ in potential pathogens. Recent studies highlight the environmental advantages of "green" synthesis over traditional methods of nanoparticle synthesis. Moreover biological applications of nano-particles synthesized by green chemistry are encouraged due to minimum cytotoxic qualities. In current scenario, nano-particles present an interesting substitute to currently available antibiotics to combat emergence of multi-drug resistant pathogens. (C) 2016 iGlobal Research and Publishing Foundation. All rights reserved.
\end{abstract}

Conference Proceedings: International Conference on Advances in Plant and Microbial Biotechnology (PMB2017); JIIT, Noida: February 02-04, 2017

Indo Global Journal of Pharmaceutical Sciences( ISSN 22491023 ; CODEN- IGJPAI; NLM ID: 101610675) indexed and abstracted in EMBASE(Elsevier), SCIRUS(Elsevier),CABI, CAB Abstracts, Chemical Abstract Services(CAS), American Chemical Society(ACS), Index Copernicus, EBSCO, DOAJ, Google Scholar and many more. For further details, visit http://iglobaljournal.com 